\title{
Integrated modeling in the UK: Practical usability of integrated models
}

\author{
David Simmonds \\ David Simmonds Consultancy Ltd. \\ Cambridge and Heriot-Watt University, Edinburgh \\ dcs@davidsimmonds.com
}

\begin{abstract}
This short paper reviews the range of planning issues that are currently being addressed in Britain and considers how the nature of these issues and the ways in which choices are being assessed impact the modeling approaches being adopted, in particular, by the author's own consultancy practice. The paper briefly outlines current developments in governance and analytical requirements; implications of trends toward more detailed (and more time-consuming) transport modeling; and the role of microsimulation in both research and planning practice. It concludes primarily that the practicality of model operation, particularly in terms of model run times, is of critical importance, and in many cases, determines whether major planning decisions are made on the basis of formal analysis rooted, albeit indirectly, in research, or without such a basis at all. A secondary conclusion relates to the possible use of output from detailed microsimulation models as a basis for calibrating aggregate models.
\end{abstract}

\section{Article history:}

Received: April 10, 2017

Accepted: February 6, 2018

Available online: May 10, 2019

\section{Introduction}

In this short note I review the range of planning issues that are currently being addressed in Britain and consider how the nature of these issues, and the ways in which choices are being assessed impact on the modeling approaches that my colleagues and I have adopted in our consultancy work. Our work accounts for a significant part of the integrated modeling currently being carried out in Britain and gives us a very particular view of modeling combining a need for theoretical rigour with an absolute requirement for practicality. The questions arising relate very directly to some of the key issues in current debates about which kinds of models should be used in which circumstances, and the about interfaces between land-use and transport.

\section{Context: Decisions to be made}

The planning issues at hand in Britain involve a number of very major internal transport infrastructure questions including (for example)

- major highway improvements in the Highlands of Scotland;

- major rail and road proposals (developed by different agencies) for the North of England, including the suggestion of a major tunnel through the Pennine hills between Manchester and Sheffield;

- major road and rail proposals (again developed by different agencies) for the Oxford - Milton

Copyright 2019 David Simmonds

http://dx.doi.org/10.5198/jtlu.2019.1206

ISSN: 1938-7849 | Licensed under the Creative Commons Attribution - Noncommercial License 4.0

The Journal of Transport and Land Use is the official journal of the World Society for Transport and Land Use (WSTLUR) and is published and sponsored by the University of Minnesota Center for Transportation Studies. 
Keynes - Cambridge corridor (orbital routes around London from north to west, at a radius of about $100 \mathrm{~km}$ from London)

- the HS2 high-speed rail proposal, for new lines from Leeds, Manchester and Birmingham to London;

- new crossings of the Thames, both within East London and east of the lowest existing crossing at Dartford; and

- a third cross-London rail route, Crossrail2, linking existing suburban lines north-east and south-west of London by new tunnels through central London.

All this is in the context first of the countries of Great Britain perceiving that they are economically disadvantaged by having under-invested in infrastructure of all kinds over recent decades, an issue that is being considered by a new National Infrastructure Commission, and secondly of a serious and growing crisis of housing supply and affordability. The appraisal (i.e., ex ante evaluation) of investment proposals is as a result strongly focused on economic impacts, both regional and national, and on the scope for such investment to facilitate additional housing development. Environmental impacts are not being ignored, but are generally examined in other workstreams, and are therefore not considered further here.

\section{Governance and analytical requirements}

I focus here on what is happening in England; the situations in Scotland and Wales are slightly different.

Decision-making in England has until now remained highly centralised, with nearly all major decisions ultimately determined by central government. For transport investment, this meant (and still means) a great deal of weight attached to the Department for Transport's appraisal ${ }^{1}$ guidance, now published electronically as WebTAG. ${ }^{2}$ WebTAG is the Department's interpretation of the general guidance on appraisal published by the Treasury; other Departments have different and generally less elaborate interpretations.

WebTAG covers all aspects of transport scheme appraisal including advice on the assessment of effects that cannot be quantified, or that can be quantified but not monetised. However, most attention is given to the quantified, monetised aspects of economic assessment that contribute to the calculation of a benefit-cost ratio (BCR). This starts from the assumption that if the economy is assumed to consist of perfect markets, it is sufficient to measure the benefits of a transport intervention by measuring the benefits in the transport market, because any other effects (such as land-use change) will simply be transfers or transformations of those benefits (or malefits).

Over the last decade, WebTAG has been revised to recognize that markets are not always perfect, in particular through agglomeration and related effects, and through the possibility that transport interventions may enable development of additional housing. A new version released in December $2017^{3}$ takes this process somewhat further and recognizes a role for supplementary economic modeling to consider wider effects. However, it insists that the core of WebTAG is appraisal in terms of economic welfare (i.e., including non-market effects such as savings in leisure travel time) and cautions that positive impacts in gross value added cannot be interpreted simply as gains in welfare. It also remains focussed very much on national-level benefits and makes only brief mention of the interest that local planners and politicians rightly take in appraisal at more local levels.

\footnotetext{
${ }^{1}$ UK Department for Transport terminology distinguishes between "appraisal" and "evaluation", "appraisal" being ex ante assessment of the case for a proposed intervention, and "evaluation" being ex post assessment of the costs and benefits of a completed intervention.

${ }^{2}$ See https://www.gov.uk/guidance/transport-analysis-guidance-webtag (accessed 31 March 2017)

3 See https://www.gov.uk/government/publications/webtag-forthcoming-change-to-wider-economic- impacts (accessed 3 March 2018). A draft was issued for consultation in late 2016.
} 
At the same time, and in contrast, government has been making considerable efforts to promote some devolution of decision-making and of financial responsibility for major planning decisions to city regions within England, coupled to reform of local governance involving directly elected mayors (as in London) and new (indirectly elected) bodies to whom they will be accountable. This has part of the programme of City Deals, ${ }^{4}$ whereby central government is offering the new city region authorities the opportunity to borrow large sums for local investment programmes. The criteria for these programmes are much more focussed on regional impacts in terms of gross value added rather than economic welfare. The need to assess both individual schemes (including roads, public transport, ports, water supply and management, regeneration and redevelopment, public realm (urban design) improvements, and other measures) and packages of schemes has created a demand for analysis outside the scope of WebTAG or any other departmental guidance. It has also imposed new timescales, since the time allowed for defining the investment programme for each region is often linked to purely political timescales measured in months rather than to more technical programmes taking years. This means that a whole range of possible investments has to be appraised and, in some sense, "optimized" in a shorter period than that normally allowed for the examination of a single major transport scheme.

\section{$4 \quad$ Modeling}

The models which we are using to contribute to appraisal in these different contexts are applications of the DELTA package, which has been developed over the last two decades. The broad outline of its development to date can be summarised in four phases:

- the first phase (1995-99) implementing models for the spatial distribution of households and jobs within a single city, modeling change over time and responses to both transport and planning interventions, but respecting exogenously fixed scenarios for the city as a whole (Simmonds, 1999);

- secondly (from 1999) the integration of such models into a wider regional/national economic and demographic framework, creating multi-level systems as advocated by Wegener, though still with fixed scenarios for the larger region or country (Simmonds, 2001);

- thirdly (from 2005) the use of such models to assess net economic impacts using post-model calculations to assess agglomeration economies and related impacts, effectively adding a new level of economic modeling in the appraisal process (see, Simmonds \& Feldman, 2013), and also the use of "open" versions of models for small regions in which improvements in transport and land-use conditions can attract additional jobs and residents from the wider world - implicitly treating the modeled region as part of a larger system (see, for example, Revill, Dobson, Simmonds, Dalgleish, \& Byers, 2014);

- most recently (from 2013) the incorporation of agglomeration effects into the core model design, meaning that planning and transport interventions which contribute to more efficient working of the economy bring about net growth both directly and through multiplier effects. Work continues towards further integration of land-use/transport and economic modeling, a path which others are also pursuing (see, for example, Anas \& Liu, 2007; Zhu, Jin, \& Echenique, 2012).

DELTA now supports a range of urban, regional and national models which have in common that they are deterministic (no stochastic components) and involve running a series of model components over time for each forecast. The comparison between this kind of dynamic model and other approaches was considered in Simmonds, Waddell, and Wegener (2013).

${ }^{4} \mathrm{https} / /$ www.gov.uk/government/policies/city-deals-and-growth-deals?page=3\&public_timestamp $\% 5 \mathrm{Bfrom} \% 5 \mathrm{D}=\&$ public timestamp\%5Bto\%5D= (accessed 3 March 2018) 


\section{Transport modeling in land-use/transport interaction}

Ideally, the set of model components run in each year would include the transport model in each year. In practice, transport model run times have always precluded that, and the most frequent use of a transport model has been to run it for alternate years of the forecast. In many cases, it has only been possible to run the transport model every fifth or tenth year, which gives rise to very lumpy transport impacts on landuse. Recently, there has been a marked tendency for transport models to get much slower: for example, the main strategic multi-modal model for London has recently gone from taking about 12 hours per run to taking over two days. This has a very serious impact on the ability to run full land-use/economy/ transport interaction models, especially in the (increasingly common) cases where political timetables require rapid analysis of the impacts and benefits of a wide range of alternatives, and in some cases the identification of an optimal package chosen from a long list of alternatives. "Rapid" in this context can mean that the analysis has to be done in a modest number of weeks, which in turn requires the use of a model which can be run in two or three hours, with multiple runs of the model being done in parallel on each of a number of computers.

Such a work programme clearly excludes transport models that take days (or in at least one case, a week) to run for each forecast year to which they are applied.

One consequence of this has been the extensive use of what we have named "land-use modeling influenced by transport" or LUMIT modeling, in which data is passed from a transport model to the land-use/economic model but not in the opposite direction. Possible transport interventions are therefore tested in conventional stand- alone transport modeling, and the potential feedbacks from impacts on land-use and economic activity are simply disregarded.

This is obviously not entirely satisfactory, though we would argue strongly that it is better than not considering land-use/economic impacts at all. The LUMIT approach has rightly been criticized in peer review for not considering the feedback from land- use change to transport, though the peer reviewers have not addressed the question of whether LUMIT analysis is better than no analysis. We would argue that it is. Government interest in assessing land-use/economic impacts of transport stems originally from trying to distinguish the real benefits of such impacts from the often-extravagant claims of such benefits made by those promoting particular schemes (see, for example, SACTRA, 1999). The feedback from land-use change to transport (i.e., the linkage including in LUTI but missing in LUMIT) will nearly always be a negative feedback (especially for highway schemes). If a proposal does not show significant benefits from land-use/economic impacts in a LUMIT framework, it is extremely unlikely to find any additional benefits in a LUTI analysis. LUMIT therefore provides a useful first estimate.

What we have also done to address this is to create our own "highly strategic transport model" or HSTM which can be run very quickly in the context of a LUTI model. This meant reversing a 20-yearold resolution not to get directly involved in transport modeling, in order to develop something appropriate. What we have created is a system which can be calibrated using data from a conventional multimodal transport model and which provides a broad-brush assessment of how travel times will change as a result of changing levels of congestion which may be generated by land-use change. It can also provide a broad-brush assessment of the impacts of new transit infrastructure and services, given estimates of how these will change the generalised costs of travel between different groups of zones. It does not attempt to carry out a detailed or responsive traffic assignment: the absence of network modeling and limited iteration is essential to its high speed of operation ( 5 to 10 minutes per run, depending on the application $^{5}$ ). The estimates of congestion change rely on area speed-flow relationships, i.e., calculating

\footnotetext{
${ }^{5}$ Overall times for full LUTI modeling including HSTM at five-year intervals are 40 to 50 minutes per decade of forecast, meaning that a 20- or 25-year forecast can be run in less than two hours, and that several rounds of testing can be carried out each working day. With this kind of turnaround, the time taken to analyze the results and to define and set up the next round of tests starts to become more of a constraint than model run time.
} 
changes in travel time from changes in the volume of traffic flowing through each zone rather than from the changes in traffic allocated to explicit links (highways) and nodes (intersections). In this and other respects the model is in the long-standing tradition of "highly strategic" transport models (see, for example, Oldfield, 1993; Bates, Brewer, Hanson, McDonald, \& Simmonds, 1991; Roberts \& Simmonds, 1997). Travel speeds are critical to changes in productivity and hence to net economic impact.

\section{Microsimulation of land-use change}

The use of microsimulation in the practical application of LUTI modeling is increasingly standard practice in academic research but remains problematical in planning practice. Some ten years ago we developed (for the UK Department for Transport) a microsimulation version of most of the household/ person modeling components of DELTA (Feldman, Mackett, Richmond, Simmonds, \& Zachariadis, 2010). This was microsimulation only in its representation of agents; it remained aggregate in its representation of space (and indeed the idea of parcel-level or raster-cell analysis is rarely mentioned in British planning practice except for some environmental calculations of traffic impacts). Our experience with SimDELTA confirmed what is well-known: that microsimulation can offer great advantages in terms of the flexibility of adding extra variables and applying sophisticated choice procedures, plus the scope to correctly explain the workings of the model in intuitive terms of what happens to, and what choices are made by, modeled households and individuals.

Those advantages are diminished if the project does not have the resources to synthesise or to forecast the additional variables or to deal with unexpected complexities in the data; but more importantly, Monte Carlo microsimulation (which for practical modeling purposes is microsimulation ${ }^{6}$ ) raises major practical problems, many of which have been raised by Professor Michael Wegener ${ }^{7}$. Some of the lessdiscussed difficulties arise acutely in model testing, in that the random element can make it difficult to reproduce problems in order to test that they have been resolved. Better known is that the stochastic variation in results makes it necessary to run models repeatedly, and to undertake some kind of averaging of results over numerous different model runs in order to average at a "single figure" estimate for a particular result, especially where the required result is the difference in results to show the impact of a particular intervention. This can in moderate cases limit the level of detail at which the results can be analyzed, thereby undermining some of the intended advantages of microsimulation (see, for example, Moeckel, 2007) Even if microsimulation models were faster than deterministic models, the number of repetitions would make their use impractical ${ }^{8}$ — especially bearing in mind that in the majority of cases, the interventions that have to be examined directly affect only a small part of the system being modeled. ${ }^{9}$ Even where interventions are substantial, the appraisal and approval process typically involve testing relatively detailed variations on the core proposal: for example, a recent analysis of a major urban rail investment required additional tests on the effect of including or excluding some relatively minor (noninterchange) stations along the route. Likewise, the major investment package assessed for the City Deal programme typically consist of a mixture of different investments of different scales, which have to be

\footnotetext{
${ }^{6}$ We appreciate that it is possible to build aggregate models that incorporate explicit stochastic components, and to build microsimulation models that are strictly deterministic. However, such models are uncommon. In addition, even if the operation of a microsimulation model can be made deterministic, there is very often a Monte Carlo element to the synthesis of the population on which the model operates.

${ }^{7}$ See in particular Wegener (2009).

${ }^{8}$ A general but largely untested assumption is that some tens of runs are needed. However, more rigorous analysis on transport models by Rasouli and Timmermans (2013) suggested that hundreds or thousands of runs may be needed to get specific results to a level suitable for policy analysis.

${ }^{9}$ It is noticeable that much of the use of the IRPUD model (the most successful LUTI model to incorporate significant use of Monte Carlo microsimulation) has been to test city-wide strategies aimed at reduction in greenhouse gas emissions and at other environmental objectives, and not at the testing of spatially specific interventions within the city.
} 
considered individually as well as jointly. Methods do exist for eliminating "noise" due to arbitrary effects of the pseudo-number generation (see, for example, Simmonds, Christodoulou, Feldman, \& McDonald, 2011) but this does not overcome the fundamental problem of stochastic variation in results.

This is not to deny the intellectual attraction of microsimulation and of being able (if large numbers of repeated model runs can be carried out) to quantify the uncertainty attaching to forecasts. (Whether the processes of political and public debate can make good use of such information is debateable; consideration of major investment decisions in Britain has been hampered by a tendency of the media to latch on to the largest numbers quoted in any particular document.) What we suggested at the conclusion of the SimDELTA project and hope to pursue further is that it may be possible to use large-scale Monte Carlo microsimulation models, carefully developed and calibrated, and run through large numbers of simulations for appropriate ranges of inputs, as a means of generating "synthetic data" on which conventional and much simpler aggregate models could be calibrated. This could perhaps help to address some of the issues arising in calibrating models on observed data, such as (for example) "treatment bias" - the risk that estimates of the impact of new transport infrastructure or services are biased because new infrastructure and services are more likely to be built to serve some kinds of locations (e.g., rapidly growing ones) than others; it would be possible in a microsimulation model to generate synthetic data for the impacts of randomly chosen projects.

The one application of this idea which we have been able to pursue to date is the use of synthetic data output from SimDELTA on household changes over time, resulting from a detailed simulation of demographic changes to individuals and of processes such as couple formation or dissolution, as the basis for a simpler Markov-chain like model of household transitions in DELTA. In SimDELTA the household changes are the result of a range of changes to individual (aging, giving birth, dying, forming a household with a partner or splitting from him/her) which were simulated with fairly elaborate sets of context-dependent probabilities (e.g., age-dependent survival rates) built up from statistics of population registration and other sources. In a typical working DELTA model, the coefficients are for example the probability that in a given year a young single household will become part of a couple, or that a young couple will become a couple with a child or children. This is a relatively robust example, in that the both model assume that the demographic changes are independent of the other aspect of the scenarios and interventions being tested; the process of using the microsimulation results is therefore a straightforward one of allocating each microsimulated household (itself defined by the number and characteristics of the members of the household, e.g., a working male and a retired female, both aged 62) to the appropriate one of the aggregate model household categories (defined by the mix of members, e.g., two or more adults, at least one working, and no children) and estimating what proportion of households transform from one category to another in an average year. Using microsimulation outputs to calibrate the model for response to policy variables would raise more complex questions about the range of interventions or other input changes that would need to be tested in the microsimulation in order to provide a valid dataset for the aggregate model calibration - although, as already noted, one of the attractions of this idea is that it would be potentially possible to microsimulate a much wider range of interventions than can be observed in practice for any one region.

The possibility of other applications of using synthetic results from research-oriented microsimulation models to calibrate aggregate models for use in practice is one that we hope to pursue further with researchers developing and operating appropriate microsimulation models.

\section{Conclusions}

The conclusion of this short paper is simply that the practicalities of model implementation and operation are critically important in determining whether formal land-use/transport models are to be used to 
contribute to major planning decisions.

There are some cases in which the timescale of public decision-making is such as to allow sufficient time for model development and application without imposing particular constraints on the form of model; but many more cases in which the time available to assemble analytical evidence and to carry out appraisals is politically determined and very short, and the analytical choice is between using a model which can be run quickly (possibly to test a large number of options) or not using a model at all. The author is very much of the view that in these circumstances well-designed and appropriate models (and associated appraisal methods) that can be run quickly are better than no model at all. These fast models are then likely to be quite different from state-of-the-art research models but should draw on the results of such research. Calibrating fast, aggregate models to the results of much more detailed but slower microsimulation models would be one way to achieve this.

\section{Acknowledgements}

This note has been developed from a presentation given to the TUM symposium on Integration of Land-Use and Transport Models, November 2016. The author is grateful to Professor Rolf Moeckel for the invitation to take part in the symposium, to all the other participants for the stimulating discussion during the meeting, and to the anonymous JTLU reviewers for helpful comments.

The developments of DELTA and SimDELTA, and their application to the projects mentioned, have been carried out through the enthusiasm and hard work of colleagues at David Simmonds Consultancy and elsewhere. The author is profoundly grateful for all their contributions and takes full responsibility for any errors or misinterpretations in this note. All opinions are those of the author and not of client organizations. 


\section{References}

Anas, A., \& Liu, Y. (2007). A regional economy, land use, and transportation model (RELU-TRAN): Formulation, algorithm design and testing. Journal of Regional Science, 47, 415-455.

Bates, J., Brewer, M., Hanson, P., McDonald, D., \& Simmonds, D. C. (1991). Building a strategic model for Edinburgh. Proceedings of Seminar D, PTRC 19th Summer Annual Meeting. London: PTRC.

Feldman, O., Mackett, R., Richmond, E., Simmonds, D., \& Zachariadis, V. (2010). A microsimulation model of household location. In F. Pagliara, J. Preston, \& D. Simmonds (Eds.) Residential location choice: Models and applications. Berlin: Springer-Verlag.

Moeckel, R. (2007). Simulation of firms to discover planning policies reducing urban sprawl of jobs. Paper presented to the 10th International Conference on Computers in Urban Planning and Urban Management (CUPUM). Retrieved from https:/www.msm.bgu.tum.de/fileadmin/w00bvh/www/ publications/2007_moeckel_c upum.pdf

Oldfield, R. H. (1993). A strategic transport model for the London area (Research Report 376). Crowthorne, UK: Transport Research Laboratory.

Rasouli, S., \& Timmermans, H. (2013). Uncertainty in complex model systems. Presentation to MOEBIUS Conference, CEPS/INSTEAD, Esch-sur-Alzette (Luxembourg), October $2013 . \quad$ Retrieved from http://mobil-t.ceps.lu/fichiers/2013_MOEBIUS_conference/RasouliTimmermans_ Uncertainty_in_complex_model_systems.pdf

Revill, E., Dobson, A., Simmonds, D., Dalgleish, S., \& Byers, N. (2014). FLUTE: The application of a land-use and transport model to prioritize infrastructure investment. Paper presented to the European Transport Conference, Frankfurt, September 28-October 1, 2014. Retrieved from www. etcproceedings.org

Roberts, M., \& Simmonds, D. C. (1997). A strategic modelling approach for land-use policy development. Paper presented to the World Conference on Transport Research, Sydney, Australia, 1995. Traffic Engineering and Control, 38, 377-384.

SACTRA (Standing Advisory Committee on Trunk Road Assessment). (1999). Transport and the economy. London: HMSO.

Simmonds, D. C. (1999). The design of the DELTA land-use modelling package. Environment and Planning B: Planning and Design, 26(5), 665-684.

Simmonds, D. C. (2001). The objectives and design of a new land-use modelling package: DELTA. In G. Clark \& M. Maden (Eds.) Regional science in business (pp. 159-188). Berlin: Springer-Verlag.

Simmonds, D. C., Christodoulou, A., Feldman, O., \& McDonald, M. (2011). Latest developments in the SimDELTA model: Investigation of stochastic variation and development of a disaggregate car ownership model. Paper presented to the 12th International Conference on Computers in Urban Planning and Urban Management (CUPUM), Lake Louise, Alberta, July 2011. Retrieved from https://www.davidsimmonds.com/publications

Simmonds, D. C., \& Feldman, O. (2013). Modelling the economic impacts of transport changes: Experience and issues. In F. Pagliara, M. de Bok, D. Simmonds \& A. Wilson (Eds.) Employment location in cities and regions: Models and applications. Heidelberg: Springer-Verlag. 